\title{
Short-Range Spin Glasses: Results and Speculations
}

\author{
C. M. Newman* \\ newman e cims . nyu .edu \\ Courant Institute of Mathematical Sciences \\ New York University \\ New York, NY 10012, USA
}

\author{
D. L. Stein ${ }^{\dagger}$ \\ dls @ physics.arizona.edu \\ Depts. of Physics and Mathematics \\ University of Arizona \\ Tucson, AZ 85721, USA
}

\begin{abstract}
This paper is divided into two parts. The first part concerns several standard scenarios for how short-range spin glasses might behave at low temperature. Earlier theorems of the authors are reviewed, and some new results presented, including a proof that, in a thermodynamic system exhibiting infinitely many pure states and with the property (such as in replica-symmetrybreaking scenarios) that mixtures of these states manifest themselves in large finite volumes, there must be an uncountable infinity of states.

In the second part of the paper, we offer some conjectures and speculations on possible unusual scenarios for the low-temperature phase of finite-range spin glasses in various dimensions. We include a discussion of the possibility of a phase transition without broken spin-flip symmetry, and provide an argument suggesting that in low dimensions such a possibility may occur. The argument is based on a new proof of Fortuin-Kasteleyn random cluster percolation at nonzero temperatures in dimensions as low as two. A second speculation considers the possibility, in analogy to certain phenomena in Anderson localization theory, of a much stronger type of chaotic temperature dependence than has previously been discussed: one in which the actual state space structure, and not just the correlations, vary chaotically with temperature.
\end{abstract}

KEY WORDS: spin glass; Edwards-Anderson model; Sherrington-Kirkpatrick model; replica symmetry breaking; mean-field theory; pure states; metastates; interface; Fortuin-Kasteleyn; random cluster percolation; Anderson localization

${ }^{*}$ Partially supported by the National Science Foundation under grant DMS-01-02587.

${ }^{\dagger}$ Partially supported by the National Science Foundation under grant DMS-01-02541. 


\section{Introduction}

In this paper we consider Ising spin glass models, in particular the infinite-range SherringtonKirkpatrick (SK) model [1] and the nearest neighbor Edwards-Anderson (EA) model [2] on $\mathbf{Z}^{d}$. The SK Hamiltonian (for $N$ spins) is

$$
\mathcal{H}_{N}=-(1 / \sqrt{N}) \sum_{1 \leq i<j \leq N} J_{i j} \sigma_{i} \sigma_{j}
$$

where the couplings $J_{i j}$ are independent, identically distributed random variables chosen, e.g., from a Gaussian distribution with zero mean and variance one.

In a series of papers, Parisi and collaborators [3, 4, 5, 6] proposed, and worked out the consequences of, an extraordinary ansatz for the nature of this phase. Following the mathematical procedures underlying the solution, it came to be known as replica symmetry breaking (RSB). The starting point of the Parisi solution was that the low-temperature spin glass phase comprised not just a single spin-reversed pair of states, but rather "infinitely many pure thermodynamic states" [4], not related by any simple symmetry transformations.

What is primarily of interest is the distribution of the overlaps between two SK 'pure states' [7] $\alpha$ and $\beta$, defined as

$$
q_{\alpha \beta}=\frac{1}{N} \sum_{i=1}^{N}\left\langle\sigma_{i}\right\rangle_{\alpha}\left\langle\sigma_{i}\right\rangle_{\beta},
$$

where $\langle\cdot\rangle_{\alpha}$ is a thermal average in pure state $\alpha$, and dependence on the coupling realization $\mathcal{J}$ and temperature $T$ has been suppressed. The overlap distribution is constructed by choosing, at fixed $N$ and $T$, two of the pure states $\alpha$ and $\beta$ present with probabilites $W_{\alpha}$ and $W_{\beta}$ in the Gibbs state. The probability that their overlap lies between $q$ and $q+d q$ is then given by the quantity $P_{\mathcal{J}}(q) d q$, where

$$
P_{\mathcal{J}}(q)=\sum_{\alpha} \sum_{\beta} W_{\mathcal{J}}^{\alpha} W_{\mathcal{J}}^{\beta} \delta\left(q-q_{\alpha \beta}\right)
$$

As before, we suppress the dependence on $T$ and $N$ for ease of notation. The average $P(q)$ of $P_{\mathcal{J}}(q)$ over the disorder distribution is commonly referred to as the Parisi overlap distribution, and serves as an order parameter for the SK model.

The EA Hamiltonian in zero external field is given by

$$
\mathcal{H}=-\sum_{\substack{x, y \\|x-y|=1}} J_{x y} \sigma_{x} \sigma_{y},
$$

where the nearest-neighbor couplings $J_{x y}$ are defined in exactly the same way as the $J_{i j}$ in the SK Hamiltonian (1). In this model, and unlike in the SK model, thermodynamic pure, mixed, 
and ground states are standard, well-defined (see, e.g., [8, 9]) objects, constructed according to well-established prescriptions of statistical mechanics [10, 11, 12, 13, 14, 15, 16].

Now suppose - as has often been conjectured for finite-range spin glasses [17, 18] — that there are many pure states not simply related to each other by symmetry transformations. If this is the case, it was shown in [19] that local variables, such as correlations, will vary chaotically and unpredictably as volume size changes (with, say, periodic boundary conditions on each volume). Is it then even possible to describe the nature of large finite volume systems via a single infinite volume object, and if so, how? It turns out that an object, called the metastate [20] by the authors, can be constructed to capture the nature of the behavior inside finite systems as volume increases. The metastate is a useful tool that describes the empirical distribution of local variables as volume increases without bound, and is described in more detail in the companion paper [21] and in [8, 20, 22, 23, 24, 25]. The seemingly chaotic behavior with increasing volume is modelled by random sampling from the metastate, regarded as a probability measure on the space of Gibbs states. Using the metastate approach, we now examine more closely the nature of the low-temperature spin glass phase.

\section{The trinity of scenarios}

There are three scenarios that have received the major share of attention in the current literature. Here we refer only to those dealing with the pure state structure of the low-temperature spin glass phase. Other pictures have also received a great deal of attention, particularly the excited state scenario of Krzakala-Martin [26] and Palassini-Young [27]. Proving or disproving these or related pictures is important for achieving a thorough understanding of the low-temperature physics of spin glasses. However, because it can be shown [28, 29] that these pictures describe excitations that do not alter pure state structure, their presence or absence can be logically incorporated into any of the three pictures that we are about to describe (although on heuristic grounds they appear incompatible with the RSB picture).

Low-temperature pictures of spin glass long-range order and broken symmetry start with an assumption about the number of pure states $\mathcal{N}(\beta, d)$ (which is the same for almost every coupling realization $\mathcal{J}$ - see, e.g., [22]). They assume also a putative critical (inverse) temperature $\beta_{c}(d)<$ $\infty$ separating a paramagnetic phase for $\beta<\beta_{c}$ from a spin glass phase for $\beta>\beta_{c}$. Although there is good numerical [17, 30, 31, 32] and some analytical [33, 34] evidence that above some lower critical dimension $d_{c}^{l}$ there does exist such a finite $\beta_{c}(d)$, there is as yet no proof or even a strong physical argument supporting such a conjecture. Moreover, there is no logical reason why 
there cannot be two or more phase transitions in some dimensions. However, we will not attempt to enumerate all possibilities here; we will confine ourselves to the most likely scenarios. We defer to Sec. 因 a consideration (mostly for the fun of it) of some of the more outlandish sounding possibilities.

The actual value of $\mathcal{N}(\beta, d)$ is not rigorously known at large $\beta$ for any $d \geq 2$. There does exist a rigorous argument [35] supporting — but not completely proving - the conjecture that $\mathcal{N}(\infty, 2)=2$, and a heuristic argument [36] supporting the conjecture that $\mathcal{N}(\beta<\infty, 2<$ $d<8) \leq 2$. It is generally assumed (but also not proved) that spin-flip symmetry is broken for $\beta_{c}<\beta<\infty$, so that pure states come in global spin-flip reversed pairs.

Given all these assumptions, pure state scenarios generally assume either a single pair of pure states or infinitely many. Again, there is at this time no argument proving that one cannot have, say, $\mathcal{N}(\beta, d)=20$; it's just difficult to imagine why such a scenario should occur. There is, however, a reasonably strong heuristic argument [24] indicating that if $\mathcal{N}(\beta, d)=\infty$, it must be an uncountable infinity (we will prove this in Sec. 3 for the RSB picture).

While an assumption about $\mathcal{N}(\beta, d)$ is the starting point for each of the three pictures we now describe, they are each much more than a simple assertion about the number of pure states. In particular, there are potentially many 2-state or many-state pictures that correspond to none of these three scenarios. Here the only aspect of the three on which we will focus is the relationship among the pure states.

\subsection{Heuristic Description}

The scenarios are, in order of increasing complexity, the scaling/droplet (SD) picture [37, 38, 39. 40, 41, 42, 43], the chaotic pairs (CP) picture [8, 9, 19, 20, 23, 24], and the RSB picture [3, 4, 5, 6, [18. The first is a 2-state scenario, while the second and third are many-state scenarios. The pure state structure in these pictures is normally described through the Parisi overlap function $P(q)$ [18]. This function needs to be used with great care in describing pure state structure in short-range models; see [8, 24, 41] for a discussion of some of the pitfalls and problems that can occur in its applications.

In the next subsection we provide precise definitions of the pure state structure of these pictures; we limit ourselves here to brief heuristic descriptions. As already noted, scaling/droplet is a twostate picture. The overlap distribution is therefore simply a pair of $\delta$-functions at $q= \pm q_{E A}$, where $q_{E A}$ is the Edwards-Anderson order parameter [2], regardless of whether replicas and overlaps are taken before or after the thermodynamic limit is taken, as shown in Fig. 1 (see below and 

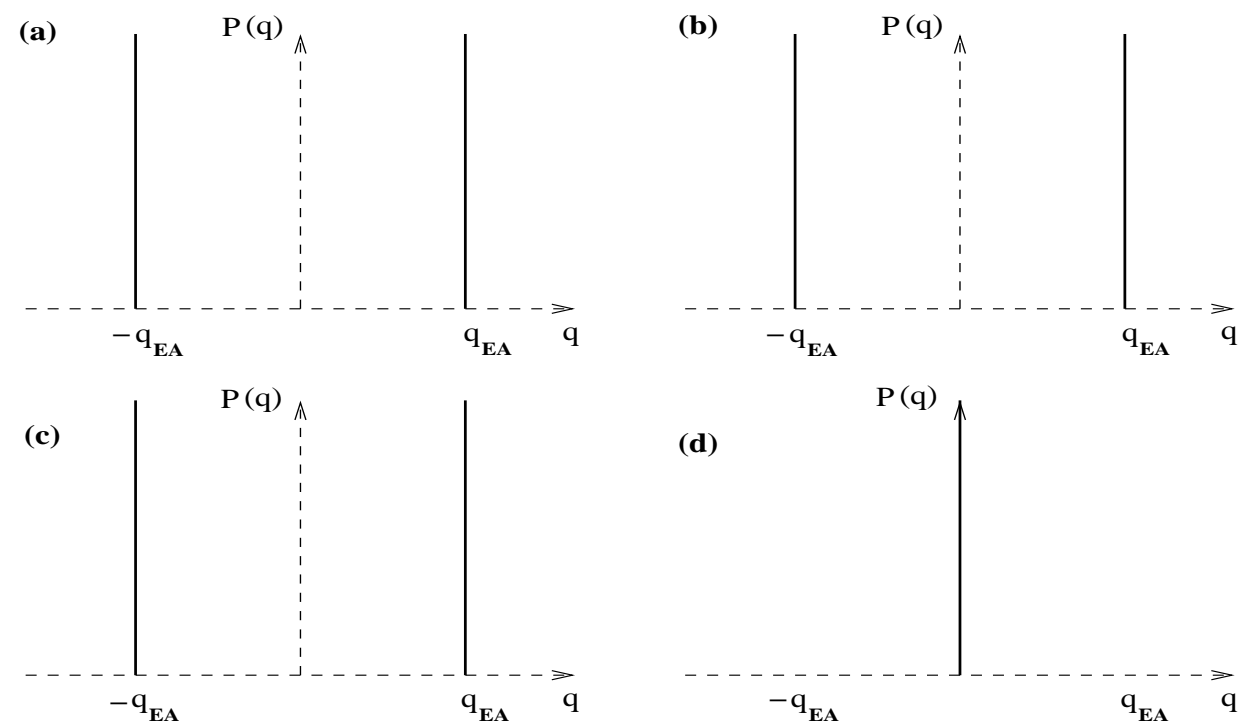

Figure 1: (From Ref. [9].) The spin overlap function $P(q)$ at $T<T_{c}$ for: (a) a two-state picture when replicas are taken before the thermodynamic limit; (b) the many-state chaotic pairs picture when replicas are taken before the thermodynamic limit; (c) a two-state picture when replicas are taken after the thermodynamic limit; (d) the many-state chaotic pairs picture when replicas are taken after the thermodynamic limit (conjectured).

especially [8, 9, 22] for a more detailed discussion of the difference between the two procedures).

The CP picture considers the possibility of an infinite number of pure state pairs, but with a trivial overlap structure: all large, finite volumes would display an overlap structure equivalent to the SD picture because only a single pair of pure states appears in each of these volumes. The actual pure state pair, however, varies chaotically as volume changes because different volumes select different members from the infinite ensemble of such pairs. The overlap distribution for the infinite volume in the $\mathrm{CP}$ picture, constructed by choosing replicas and taking their overlaps after going to the thermodynamic limit, would then presumably be a simple $\delta$-function at zero overlap (cf. Fig. 1).

There are actually two RSB pictures, which we have called 'standard' and 'nonstandard' SK [20, 23, 24, 44]. The first chooses replicas after taking the thermodynamic limit, and the second before. Both have nontrivial overlap structures, which will be described in the next subsection.

Before turning to that, we need to say a few words about the dimension dependence of the three pictures. The only one of these with specific predictions is the SD picture, which asserts that in every finite dimension where spin-flip symmetry is broken, there is only a single pure state pair. The $\mathrm{CP}$ picture does not make any corresponding claim; it merely asserts that if $\mathcal{N}(\beta, d)=\infty$ at 
some $(\beta, d)$, then the overlap structure must be trivial in the manner specified above. If the RSB picture correctly describes the EA model at $d=\infty$, then both the SD and CP models agree that the upper critical dimension $d_{c}^{u}=\infty$ for the EA model.

The RSB picture is slightly less vague about its dimension-dependence; it does apparently assume that there exists a strictly finite $d_{c}^{u}$ for the EA model, and that replica symmetry is broken in the nontrivial manner it specifies for all $d \geq d_{c}^{u}$. The precise value of $d_{c}^{u}$ within this picture remains uncertain, but it seems to be higher than two and less than or equal to three [45, 46].

\subsection{Description via the Metastate}

We now turn to a precise description of the three competing pictures, which is greatly facilitated by using the metastate.

In SD, there is only a single pair of pure states, and these are the same in every large volume; the overlap distribution function $P_{\mathcal{J}}^{L}(q)$ in a volume $\Lambda_{L}$ therefore simply approximates a sum of two $\delta$-functions at $\pm q_{E A}$, as shown in Fig. 1(a). In the CP picture, each finite-volume Gibbs state $\rho_{\mathcal{J}}^{L}$ will still be approximately a mixture of a single pair of spin-flip-related pure states, but now the pure state pair will vary chaotically with $L$. Then for each $\Lambda_{L}, P_{\mathcal{J}}^{L}(q)$ will again approximate a sum of two $\delta$-functions at $\pm q_{E A}$.

So chaotic pairs resembles the scaling/droplet picture in finite volumes, but has a very different thermodynamic structure. It is a many-state picture, but differs from the RSB picture (see below) in that only a single pair of spin-reversed pure states $\rho_{\mathcal{J}}^{\alpha_{L}}, \rho_{\mathcal{J}}^{\overline{\alpha_{L}}}$, appears in a large volume $\Lambda_{L}$ with symmetric boundary conditions, such as periodic. In other words, for large $L$, one finds that

$$
\rho_{\mathcal{J}}^{(L)} \approx \frac{1}{2} \rho_{\mathcal{J}}^{\alpha_{L}}+\frac{1}{2} \rho_{\mathcal{J}}^{\overline{\alpha_{L}}}
$$

and the pure state pair (of the infinitely many present) appearing in a particular finite volume $\Lambda_{L}$ depends chaotically on $L$. That is, the periodic b.c. metastate is dispersed over many $\Gamma$ 's (in fact, an uncountable infinity, as we shall see below; this also occurs in the RSB picture), but (unlike in RSB) each $\Gamma$ is a trivial mixture of the form $\Gamma=\Gamma^{\alpha}=\frac{1}{2} \rho_{\mathcal{J}}^{\alpha}+\frac{1}{2} \rho_{\mathcal{J}}^{\bar{\alpha}}$. The overlap distribution for each $\Gamma$ is the same: $P_{\Gamma}=\frac{1}{2} \delta\left(q-q_{E A}\right)+\frac{1}{2} \delta\left(q+q_{E A}\right)$. It is interesting to note that there is a spin glass model (the 'highly disordered model' [47, 48, 49]) that appears to display just this behavior in its ground state structure above eight dimensions.

We now turn to the standard and nonstandard mean-field-like scenarios. The standard SK picture is perhaps most concisely described in the introduction of [50]. It requires a Gibbs equilibrium 
measure $\rho_{\mathcal{J}}(\sigma)$ which is decomposable into many pure states $\rho_{\mathcal{J}}^{\alpha}(\sigma)$ :

$$
\rho_{\mathcal{J}}(\sigma)=\sum_{\alpha} W_{\mathcal{J}}^{\alpha} \rho_{\mathcal{J}}^{\alpha}(\sigma)
$$

In this picture replicas are taken after going to the thermodynamic limit. That is, one chooses $\sigma$ and $\sigma^{\prime}$ from the product distribution $\rho_{\mathcal{J}}(\sigma) \rho_{\mathcal{J}}\left(\sigma^{\prime}\right)$, and then the overlap can be defined as

$$
Q=\lim _{L \rightarrow \infty}\left|\Lambda_{L}\right|^{-1} \sum_{x \in \Lambda_{L}} \sigma_{x} \sigma_{x}^{\prime}
$$

where $\left|\Lambda_{L}\right|$ is the volume of the cube $\Lambda_{L}$.

Suppose that $\sigma$ is drawn from pure state $\rho_{\mathcal{J}}^{\alpha}$ and $\sigma^{\prime}$ from $\rho_{\mathcal{J}}^{\gamma}$. Then (7) equals its thermal mean [44]

$$
q_{\mathcal{J}}^{\alpha \gamma}=\lim _{L \rightarrow \infty}\left|\Lambda_{L}\right|^{-1} \sum_{x \in \Lambda_{L}}\left\langle\sigma_{x}\right\rangle_{\alpha}\left\langle\sigma_{x}\right\rangle_{\gamma}
$$

and so the overlap distribution $P_{\mathcal{J}}(q)$ is given by

$$
P_{\mathcal{J}}(q)=\sum_{\alpha, \gamma} W_{\mathcal{J}}^{\alpha} W_{\mathcal{J}}^{\gamma} \delta\left(q-q_{\mathcal{J}}^{\alpha \gamma}\right)
$$

According to this picture, the $W_{\mathcal{J}}^{\alpha}$ 's and $q_{\mathcal{J}}^{\alpha \gamma}$ 's are non-self-averaging quantities, except when $\alpha=\gamma$ or its global flip, where $q_{\mathcal{J}}^{\alpha \gamma}= \pm q_{E A}$. The average $P(q)$ of $P_{\mathcal{J}}(q)$ over the disorder distribution $\nu$ of the couplings is then a mixture of two delta-function components at $\pm q_{E A}$ and a continuous part between them.

There is a technical problem (caused by chaotic size dependence [19]) in the construction of $\rho_{\mathcal{J}}(\sigma)$ that can be overcome by using the periodic b.c. metastate $\kappa_{\mathcal{J}}^{\mathrm{PBC}}$ (in fact, any couplingindependent metastate would do). One can construct [44] a state $\rho_{\mathcal{J}}(\sigma)$ which is the average over the metastate:

$$
\rho_{\mathcal{J}}(\sigma)=\int \Gamma(\sigma) \kappa_{\mathcal{J}}(\Gamma) d \Gamma
$$

One can also think of this $\rho_{\mathcal{J}}$ as the average thermodynamic state, $N^{-1}\left(\rho_{\mathcal{J}}^{\left(L_{1}\right)}+\rho_{\mathcal{J}}^{\left(L_{2}\right)}+\ldots, \rho_{\mathcal{J}}^{\left(L_{N}\right)}\right)$, in the limit $N \rightarrow \infty$. It can be proved [22, 25] that $\rho_{\mathcal{J}}(\sigma)$ is a Gibbs state.

Numerically one constructs overlaps without constructing Gibbs states at all. Such a construction (similar to that above) is described in [44], and leads ultimately to the same conclusion.

But this picture can be rigorously ruled out for the EA model [44], as will be shown in Sec. 3 ] So the most natural (and usual) interpretation of a mean-field-like picture cannot be applied to short-range spin glasses. The question then becomes: are there alternative, less straightforward interpretations? To address this question, we constructed in [20] (see also [8, 9, 22, 23, 24]) 



Figure 2: (From Ref. [8].) The overlap distribution, at fixed $\mathcal{J}$, in two different volumes $\Lambda_{1}$ and $\Lambda_{2}$ in the nonstandard SK picture.

an alternative mean-field-like picture, the 'nonstandard SK model', which we hereafter refer to simply as the RSB picture. This picture clarifies as well how broken replica symmetry should be interpreted for the SK model.

Consider again the PBC metastate, although, as always, almost any other coupling-independent metastate will suffice. The RSB picture assumes that in each volume $\Lambda_{L_{i}}$, the finite-volume Gibbs state $\rho_{\mathcal{J}, L_{i}}$ is well approximated deep in the interior by a mixed thermodynamic state $\Gamma^{\left(L_{i}\right)}$, decomposable into many pure states $\rho_{\alpha_{L_{i}}}$ :

$$
\Gamma^{\left(L_{i}\right)}=\sum_{\alpha_{L_{i}}} W_{\Gamma^{\left(L_{i}\right)}}^{\alpha_{L_{i}}} \rho_{\alpha_{L_{i}}}
$$

where explicit dependence on $\mathcal{J}$ is suppressed.

As in the chaotic pairs picture, the mixed states $\Gamma^{\left(L_{i}\right)}$ change in some "chaotic" fashion with $L_{i}$. Furthermore, each mixed state $\Gamma^{\left(L_{i}\right)}$ is presumed to have a nontrivial overlap distribution

$$
P_{\Gamma^{\left(L_{i}\right)}}=\sum_{\alpha_{L_{i}}, \beta_{L_{i}}} W_{\Gamma^{\left(L_{i}\right)}}^{\alpha_{L_{i}}} W_{\Gamma^{\left(L_{i}\right)}}^{\beta_{L_{i}}} \delta\left(q-q_{\alpha_{L_{i}} \beta_{L_{i}}}\right)
$$

of the form shown in Fig. 2. Moreover, the distances among any three pure states within a particular $\Gamma$ are assumed to be ultrametric [18].

We conclude with a brief note about zero temperature. In each $\Lambda_{L}$ (with periodic b.c.'s) there can only be a single ground state pair at $T=0$ (because we are considering Gaussian couplings rather than a $\pm J$ model). If scaling/droplet holds, then this pair is the same for all large $L$; if 
infinitely many ground state pairs exist, then the pair changes chaotically with $L$. In this respect the behavior of $\mathrm{CP}$ is the same at both zero and nonzero temperatures (below $T_{c}$ ), and the same can be said for SD. So the overlap functions of CP and RSB differ only at positive temperature: the mean-field RSB picture at $T>0$ has the $\Gamma^{(L)}$ in each volume exhibiting a nontrivial mixture of pure state pairs as in (11), while in chaotic pairs the $\Gamma^{(L)}$ appearing in any $\Lambda_{L}$ consists of a single pure state pair, as in (5). There remains, however, an important difference between the $\mathrm{CP}$ and RSB pictures at zero temperature, in that the space-filling interfaces between ground states should have energies that scale differently; see [8] and the companion paper in this volume [21] for a more detailed discussion.

\section{Replica Symmetry Breaking for Short-Range Models}

We begin by eliminating the standard SK picture from further consideration; the detailed rigorous proof appears in [44]. Using the torus-translation symmetry of the periodic b.c.'s, one can show that the Gibbs state $\rho_{\mathcal{J}}(\sigma)$ is translation-covariant; that is, $\left.\rho_{\mathcal{J}} a\right)=\rho_{\mathcal{J}}\left(\sigma^{-a}\right)$, or in terms of correlations, $\left\langle\sigma_{x}\right\rangle_{\mathcal{J}^{a}}=\left\langle\sigma_{x-a}\right\rangle_{\mathcal{J}}$, and similarly for $n$-point correlations. Translation covariance of $\rho_{\mathcal{J}}$ immediately implies, via Eqs. (7)-(9), translation invariance of $P_{\mathcal{J}}$. But, given the translation-ergodicity of the underlying disorder distribution $\nu$, it immediately follows that $P_{\mathcal{J}}(q)$ is self-averaging, and equals its distribution average $P(q)$ for a.e. $\mathcal{J}$. The impossibility of a non-self-averaging $P_{\mathcal{J}}(q)$ can also be shown for other coupling-independent b.c.'s, where torustranslation symmetry is absent, using methods described in [35].

This leaves nonstandard SK as a maximally allowed mean-field-type picture. Before discussing its viability, we explain why neither $\mathrm{CP}$ nor nonstandard SK remains viable if modified to have only a countable infinity of pure states. We do this first by a heuristic argument valid for both $\mathrm{CP}$ and nonstandard SK, and then by a rigorous argument valid only for nonstandard SK. The conclusion of this exercise is that both alternatives to the SD scenario of a single pure state pair require uncountably many (i.e., a continuum of) pure state pairs for a single fixed $\mathcal{J}$.

Consider $\rho_{\mathcal{J}}$, the average over the periodic b.c. metastate $\kappa_{\mathcal{J}}$. Changing from periodic boundary conditions to antiperiodic, or to any of the many other 'partially antiperiodic' boundary conditions related to periodic ones by a gauge transformation, leaves $\kappa_{\mathcal{J}}$ and consequently $\rho_{\mathcal{J}}$ unchanged [24]. On heuristic grounds, unless $\rho_{\mathcal{J}}$ is of the SD form with only a single pair of pure states, then this lack of dependence on boundary conditions should hold only if $\rho_{\mathcal{J}}$ is a uniform mixture over infinitely many pure states $\rho_{\mathcal{J}}^{\alpha}$ - i.e., the relative weights of all $\rho_{\mathcal{J}}^{\alpha}$ 's in $\rho_{\mathcal{J}}$ are equal. But that is clearly impossible if the number of $\rho_{\mathcal{J}}^{\alpha}$ 's is countably infinite. 
In the case of an SK-type picture, where the $\Gamma$ 's appearing in the metastate $\kappa_{\mathcal{J}}$ involve nontrivial weights $W_{\mathcal{J}}^{\alpha}$, one can go further and prove that nontrivial $W_{\mathcal{J}}^{\alpha}$ 's require uncountably many $\rho_{\mathcal{J}}^{\alpha}$ 's to appear in $\kappa_{\mathcal{J}}$. The argument proceeds as follows. If there are only countably many $\rho_{\mathcal{J}}^{\alpha}$ 's, then the corresponding weights (say, ordered by magnitude) in $\rho_{\mathcal{J}}$ will be measurable and translationinvariant functions of $\mathcal{J}$. By translation-ergodicity, this means that they are in fact independent of $\mathcal{J}$ and so remain the same if finitely many couplings $J_{x y}$ change by amounts $\Delta J_{x y}$. Moreover, one can consider the finitely many $\alpha$ 's corresponding to, say, the largest $k$ weights in $\rho_{\mathcal{J}}$, and then the distribution of their weights within the $\Gamma$ 's of the metastate $\kappa_{\mathcal{J}}$ is also independent of $\mathcal{J}$.

On the other hand, this leads to a contradiction, because a $\Gamma$ in the nonstandard SK metastate (see Eq. (11)) is a nontrivial mixture of pure states and their weights. By the AizenmanWehr transformation [25], a pure state $\rho_{\alpha}$ transforms to a pure state $\rho_{\alpha^{\prime}}$ under such a finite change $\mathcal{J} \rightarrow \mathcal{J}+\Delta J$, and its weight $W_{\alpha}$ within $\Gamma$ correspondingly changes according to

$$
W_{\alpha} \rightarrow W_{\alpha^{\prime}}=r_{\alpha} W_{\alpha} / \sum_{\gamma} r_{\gamma} W_{\gamma}
$$

where

$$
r_{\alpha}=\left\langle\exp \left(\beta \sum_{\langle x y\rangle} \Delta J_{x y} \sigma_{x} \sigma_{y}\right)\right\rangle_{\alpha} .
$$

For pure states $\alpha$ and $\delta$ that are not identical one can find a choice of $\Delta \mathcal{J}$, with all $\Delta J_{x y}$ small, such that $r_{\alpha} \neq r_{\delta}$. Then for all $\Gamma$ 's in which $W_{\alpha}(\Gamma)$ and $W_{\delta}(\Gamma)$ are nonzero, the ratio $W_{\alpha^{\prime}}(\Gamma) / W_{\delta^{\prime}}(\Gamma)$ is changed from $W_{\alpha}(\Gamma) / W_{\delta}(\Gamma)$ by the same factor $r_{\alpha} / r_{\delta}$, that is close to 1 . But this implies that $\mathcal{J} \rightarrow \mathcal{J}+\Delta \mathcal{J}$ will change the distribution of the weights within the metastate, leading to a contradiction. We have thus sketched the proof of:

Theorem 1. The PBC metastate $\kappa_{\mathcal{J}}$ for the EA spin glass cannot assign strictly positive probability to $\Gamma$ 's whose pure state decompositions satisfy both:

(i) $\Gamma=\sum_{\alpha} W_{\mathcal{J}}^{\alpha} \rho_{\mathcal{J}}^{\alpha}$, with not all $W_{\mathcal{J}}^{\alpha}=1 / 2$, and

(ii) over all these $\Gamma$ 's only countably many $\rho_{\mathcal{J}}^{\alpha}$ 's appear.

An immediate consequence of Theorem 1, when combined with our earlier arguments, is that the only remaining mean-field-like picture is the nonstandard SK model with a (periodic b.c.) metastate $\kappa_{\mathcal{J}}$ whose average $\rho_{\mathcal{J}}$ is supported on an uncountable infinity of pure states.

We cannot at this point rule this scenario out rigorously, but can provide a strong heuristic argument, based on a rigorous result, that makes it very unlikely. The rigorous result is the already mentioned invariance of the metastate [24]: two metastates constructed using gauge-related b.c.'s (e.g., periodic and antiperiodic, or any two randomly chosen fixed b.c.'s) are identical. This makes 
it difficult to see how any many-state picture can have a $\rho_{\mathcal{J}}$ supported on anything other than a uniform distribution of pure states. But if this did occur for a particular $\mathcal{J}$, an Aizenman-Wehr transformation suggests that the uniformity would be destroyed for a finitely different $\mathcal{J}^{\prime}$. (For detailed arguments, see [24].)

Why doesn't this argument also rule out chaotic pairs? Because in the chaotic pairs picture, as in scaling/droplet, there are in each $\Gamma^{(L)}$ only two pure states (although in CP the pair depends on $L$ ), each with weight $1 / 2$. All even correlations are the same in any pair of flip-related pure states, so, by Eqs. (13) and (14), any change in couplings leaves the weights unchanged.

We conclude that the only viable many-state scenario for short-range Ising spin glasses is the $\mathrm{CP}$ picture with an uncountable infinity of pure states. This picture has trivial replica symmetry breaking (cf. Fig. 1) and consequently a very simple overlap structure.

\section{Wild Possibilities}

In Sec. 2] we presented the most likely scenarios for the pure state structure of the spin glass phase in short-range models. One of these, the RSB picture, although a priori plausible, especially given its relevance to the SK model, was rigorously ruled out in two of its possible versions and excluded heuristically in its final remaining version. However, there are other, more exotic scenarios that might also occur but do not seem to have been considered. Here, for the sake of completeness (and perhaps a little whimsy) we suggest two. In order to keep the list of possibilities relatively constrained, we will assume in all that: a) a thermodynamic phase transition does exist above some lower critical dimension $d_{c}^{l}$, and $\mathrm{b}$ ) in a fixed dimension the low-temperature phase does not alternate among different scenarios, such as SD and CP, as temperature is lowered (although the third and most exotic scenario may be regarded as a kind of phase of extreme alternation).

\subsection{Phase transition without broken spin-flip symmetry}

As mentioned in Sec. [2] despite decades of effort and considerable numerical support [17, 30, 31, 32], there remains no proof of a phase transition in EA spin glasses. In this section we provide a proof that shows that a necessary (though not sufficient) condition for broken spin-flip symmetry at $T>0$ is satisfied in lattices with dimension as low as two (e.g., a triangular lattice). We also discuss a corresponding sufficient condition. Of course, this (minimal) symmetry-breaking is assumed in all scenarios discussed in Sec. 2, but here we speculate on the possibility of a phase transition without the appearance of multiple Gibbs states, and discuss why the presence of the 
necessary condition (for broken spin-flip symmetry) at some $(\beta, d)$ where the sufficient condition is absent suggests just such a possibility.

Although several approaches are possible, we use here the Fortuin-Kasteleyn random cluster (RC) representation [51, 52] extended to non-ferromagnetic models (see, e.g., [53]), which relates the statistical mechanics of Ising (or Potts) models to a dependent percolation problem. We let $\mathbf{E}^{d}$ denote the set of bonds $\langle x, y\rangle$ in some $d$-dimensional lattice $\mathbf{L}^{d}$, each with corresponding coupling $J_{x y}$. The RC approach introduces parameters $p_{x y} \in[0,1)$ by:

$$
p_{x y}=1-\exp \left[-\beta\left|J_{x y}\right|\right] .
$$

The RC distribution is a probability measure $\mu_{\mathrm{RC}}$ on $\{0,1\}^{\mathbf{E}^{d}}$, that is, on 0 - or 1 -valued bond occupation variables $n_{x y}$. It is one of two marginal distributions (the other being the ordinary Gibbs distribution) of a joint distribution on $\Omega=\{-1,+1\}^{\mathbf{L}^{d}} \times\{0,1\}^{\mathbf{E}^{d}}$ of the spins and bonds together, and is given (formally, in the infinite system) by

$$
\mu_{\mathrm{RC}}\left(n_{x y}\right)=Z_{\mathrm{RC}}^{-1} 2^{\#\left(\left\{n_{x y}\right\}\right)} \mu_{\mathrm{ind}}\left(\left\{n_{x y}\right\}\right) 1_{U}\left(\left\{n_{x y}\right\}\right),
$$

where $Z_{\mathrm{RC}}$ is a normalization constant, \# $\left(\left\{n_{x y}\right\}\right)$ is the number of clusters determined by the realization $\left\{n_{x y}\right\}, \mu_{\text {ind }}\left(\left\{n_{x y}\right\}\right)$ is the Bernoulli product measure corresponding to independent occupation variables with $\mu_{\text {ind }}\left(\left\{n_{x y}=1\right\}\right)=p_{x y}$, and $1_{U}$ is the indicator function on the event $U$ in $\{0,1\}^{\mathbf{E}^{d}}$ that there exists a choice of the spins $\sigma_{x}$ so that $J_{x y} n_{x y} \sigma_{x} \sigma_{y} \geq 0$ for all $\langle x, y\rangle[$ [54, 55, 56]. $U$ is the event that there is no frustration in the occupied bond configuration. Finite-volume versions of the above formulas, with specified boundary conditions, are similarly constructed.

The mapping of this formalism to ferromagnets follows from formulae (that do not hold for nonferromagnets) such as [57]

$$
\left\langle\sigma_{x} \sigma_{y}\right\rangle=\mu_{\mathrm{RC}}(x \leftrightarrow y),
$$

where $\left\langle\sigma_{x} \sigma_{y}\right\rangle$ is the usual Gibbs two-point correlation function and $\mu_{\mathrm{RC}}(x \leftrightarrow y)$ is the RC probability that $x$ and $y$ are in the same cluster. Similarly, with appropriate RC (wired) boundary conditions used for $\mu_{R C}$, one has

$$
\left\langle\sigma_{x}\right\rangle_{+}=\mu_{\mathrm{RC}}(x \leftrightarrow \infty) .
$$

It follows that, for ferromagnets, a phase transition from a unique (paramagnetic) phase at low $\beta$ to multiple infinite volume Gibbs distributions at large $\beta$ is equivalent to a percolation phase transition for the corresponding RC measure. 
For spin glasses (or other nonferromagnets) the situation is less straightforward. Now for two sites $x$ and $y$, 177 becomes

$$
\left\langle\sigma_{x} \sigma_{y}\right\rangle=\left\langle 1_{x \leftrightarrow \infty} \eta(x, y)\right\rangle_{R C} ; \eta(x, y)=\prod_{\left\langle x^{\prime} y^{\prime}\right\rangle \in \mathcal{C}} \operatorname{sgn}\left(J_{x^{\prime} y^{\prime}}\right),
$$

where $\mathcal{C}$ is any path of occupied bonds from $x$ to $y$. By the definition of $U$, any two paths $\mathcal{C}$ and $\mathcal{C}^{\prime}$ in the same cluster will satisfy $\prod_{\left\langle x^{\prime} y^{\prime}\right\rangle \in \mathcal{C}} \operatorname{sgn}\left(J_{x^{\prime} y^{\prime}}\right)=\prod_{\left\langle x^{\prime} y^{\prime}\right\rangle \in \mathcal{C}^{\prime}} \operatorname{sgn}\left(J_{x^{\prime} y^{\prime}}\right)$.

It is no longer evident that RC percolation is sufficient to prove broken spin-flip symmetry. Consider the case of a finite volume $\Lambda_{L}$ with fixed boundary conditions, i.e., a specification $\bar{\sigma}_{x}=$ \pm 1 for each $\bar{\sigma}_{x} \in \partial \Lambda_{L}$. For the ferromagnet, by first choosing all $\bar{\sigma}_{x}=+1$ and then all $\bar{\sigma}_{x}=-1$, one can change the sign of the spin $\sigma_{0}$ at the origin even as $L \rightarrow \infty$. That is, boundary conditions infinitely far away affect $\sigma_{0}$, which is a signature of the existence of multiple Gibbs distributions.

But for the EA spin glass, it is not clear whether, even in the presence of RC percolation, there exist any two sets of boundary conditions with the same effect. Although the infinite cluster in any one RC realization is unique, different RC realizations can have different paths from $0 \leftrightarrow \partial \Lambda_{L}$, leading to different signs for $\sigma_{0}$. So percolation might still allow for $\left\langle\sigma_{0}\right\rangle \rightarrow 0$ as $L \rightarrow \infty$.

However, it is easy to see that single RC percolation is at least a necessary condition for multiple (symmetry-broken) Gibbs phases. The contribution to the expectation of $\sigma_{0}$ from any finite $\mathrm{RC}$ cluster is zero: if a spin configuration $\sigma$ is consistent with a given $\mathrm{RC}$ bond realization within such a cluster, $-\sigma$ is also consistent and equally likely. As a consequence, at a $(\beta, d)$ where $\mathrm{RC}$ percolation does not hold, $\left\langle\sigma_{0}\right\rangle=0$ in infinite volume. We note that a slightly stronger version of this argument [56] proves that the transition temperature for an EA $\pm J$ (or other) spin glass, if it exists, is bounded from above by the transition temperature in the corresponding (disordered) ferromagnet.

Is there a modification of this approach that could lead to a proof of multiple Gibbs phases? One such possibility is what might be called double RC percolation. Here one expands the sample space $\Omega$ to include two independent copies of the bond occupation variables (for a given $\mathcal{J}$ configuration), and defines the variable $r_{x y}=n_{x y} n_{x y}^{\prime}$, where $n_{x y}$ and $n_{x y}^{\prime}$ are taken from the two copies. One then replaces percolation of $\left\{n_{x y}\right\}$ in the single RC case with percolation of $\left\{r_{x y}\right\}$. It is not hard to see that this would be a sufficient condition for the existence of multiple Gibbs phases (and consequently, for a phase transition).

Single RC percolation for the EA $\pm J$ Ising model in $d>2$ [55] has been proved [53]. Here we sketch the outline of a proof (simpler than the one in [53]) showing that one has single RC percolation even in $d=2$ - e.g., on the triangular lattice. This is already interesting for the following reason: because we are not aware of strong numerical evidence of multiple Gibbs states for this 
geometry, this may be considered evidence that single RC percolation can occur in spin glasses without broken spin-flip symmetry. But there is an even more interesting potential consequence, which we will discuss below.

The proof uses a standard Fortuin-Kasteleyn-Ginibre (FKG) argument [58] using correlation inequalities. Let $\mathbf{E}_{L}$ be the edge set confined entirely within a volume $\Lambda_{L}$. It can be shown that there exist probability measures $\nu_{L}$ on $\{0,1\}^{\mathbf{E}_{L}}$ such that, if $f(\{n\})$ and $g(\{n\})$ are nondecreasing real functions (that is, they do not decrease when any $\{n\} \rightarrow\left\{n^{\prime}\right\}$, where every $n_{x y}=1$ corresponds to $n_{x y}^{\prime}=1$, but $n_{x y}=0$ can correspond to either $n_{x y}^{\prime}=0$ or 1 ), then they are positively correlated:

$$
\langle f g\rangle_{\nu_{L}} \geq\langle f\rangle_{\nu_{L}}\langle g\rangle_{\nu_{L}}
$$

One measure satisfying this property is the independent product measure [59]. Consequently, the marginal distribution (i.e., averaged over the coupling (signs) in the $\pm J$ model) of satisfied bonds (i.e., using the "satisfaction" variables $\tilde{n}_{x y}=1$ (or else 0 ) if $J_{x y} \sigma_{x} \sigma_{y}>0$ ) at $\beta=0$, satisfies this property. Operationally, one can think of constructing this set by choosing the spins first through independent flips of a fair coin, and then choosing the sign of each bond in the same way. One then has independent, density-1/2 bond occupation, which percolates on the triangular lattice [60, 61].

Consider now the satisfaction variables $\tilde{n}_{b}$ for $\beta>0$. It is not hard to show that, at any such fixed $\beta$, and for any bond $b=\langle x y\rangle$,

$$
P\left(\tilde{n}_{b}=1 \mid\left\{\tilde{n}_{b^{\prime}}: b^{\prime} \neq b\right\}\right) \geq 1 / 2+\epsilon(\beta),
$$

where $\epsilon(\beta)$ is a nonnegative function of $\beta$. Eq. (21) implies that percolation of satisfied bonds at finite temperature dominates (in the FKG sense) percolation at infinite temperature. Since the $\mathrm{RC}$ variables $n_{b}$ are obtained from the satisfaction variables $\tilde{n}_{b}$ by a slight (for large $\beta$ ) dilution, it follows that at sufficiently low (but nonzero) temperature one has single RC percolation.

It is presumably the case that on the triangular lattice there is no broken spin-flip symmetry, and also only a single Gibbs state, at all nonzero temperatures. But it is worth entertaining the possibility that single, but no double RC percolation, does imply some sort of phase transition but with a single Gibbs state at all nonzero temperatures.

Let $\beta_{c}<\infty$ be the inverse RC percolation transition temperature for the EA model on the triangular lattice. Consider again the expectation of the spin at the origin, $\left\langle\sigma_{0}\right\rangle$, in a volume $\Lambda_{L}$ with plus boundary conditions. For $\beta<\beta_{c}$, the probability of the site 0 belonging to a cluster reaching the boundary is bounded from above by $c_{0}(\beta) e^{-c_{1}(\beta) L}$, where each $c_{i}(\beta)>0$ is a finite constant. Therefore $\left\langle\sigma_{0}\right\rangle \leq c_{0}(\beta) e^{-c_{1}(\beta) L}$. 
For $\beta>\beta_{c}$, however, the probability that the origin belongs to a cluster reaching the boundary is bounded away from zero as $L \rightarrow \infty$. In order for no phase transition to take place, there must be at fixed $\beta$ an almost exact cancellation between those RC realizations of 'positive' (in the product of the signs of the couplings) and 'negative' paths from the origin to the boundary. It is at least conceivable that, while $\sigma_{0} \rightarrow 0$ as $L \rightarrow \infty$, it falls off slower than exponentially perhaps as a power law in $L$. This would imply a phase transition from a paramagnetic phase at high temperature to a phase at low temperature with a unique Gibbs state, but one where two-point correlations decay as a power law: $\left\langle\sigma_{x} \sigma_{y}\right\rangle \sim|x-y|^{-\alpha(\beta)}$ as $|x-y| \rightarrow \infty$. This would be analogous to the Kosterlitz-Thouless phase transition for $2 D X Y$ models [62].

\subsection{Highly chaotic temperature dependence}

We discussed earlier [19] the correspondence between multiple Gibbs state pairs and the appearance of chaotic size dependence of correlations in an infinite sequence of volumes with, say, periodic boundary conditions. A possibly related phenomenon is the speculated presence in spin glasses (both infinite- and short-range) of chaotic temperature dependence (CTD). Roughly speaking, this refers to an erratic behavior of correlations, upon changing temperature, on lengthscales that diverge as the temperature increment goes to zero. CTD was predicted [39, 43, 63] for the EA spin glass within the SD context; it also seems to be implied by the RSB theory [64, 65, 66]. More recent numerical and analytical work (see [67] and references therein) have led to claims that CTD is not present in either the SK or the EA model (see also [68]), although [69] allows for the possibility of a weak effect at large lattice sizes. A perturbative approach [70] observes a very small effect at ninth order. At this time the issue remains unresolved. Its potential presence in spin glasses is interesting, however, and represents a qualitatively new thermodynamic feature of at least some types of disordered systems.

In this section we raise the possibility of a far stronger version of CTD, which we will call highly chaotic temperature dependence (HCTD) [36]. Unlike 'ordinary' CTD, where correlations behave in a chaotic fashion as temperature changes but the global pure state structure does not, in HCTD the number of pure states in the periodic b.c. metastate does not behave in a continuous or even monotonic fashion as temperature is lowered. This picture departs radically from any other that has appeared so far in the literature.

The HCTD scenario is summarized as follows. As in all other pictures, there exists a deterministic $T_{c}$ for a.e. $\mathcal{J}$, such that for all $T>T_{c}$ there is a unique (paramagnetic) Gibbs state. Suppose one were now to choose an arbitrary (nonzero) $T<T_{c}$. Then with probability one (i.e., for almost 
every $\mathcal{J}$ ), there is again a unique infinite volume Gibbs state (though maybe not with exponential decay of truncated correlations). Nevertheless, as temperature is lowered from $T_{c}$ to 0 for a fixed typical $\mathcal{J}$, there would be a (countably infinite) dense set of temperatures, depending on $\mathcal{J}$, with broken symmetric pure phases for that $\mathcal{J}$ at those temperatures.

Now this scenario might be dismissed, with some justification, as the authors' fevered imaginings, but it should be noted that just this sort of phenomenon does happen in other disordered systems. Consider Anderson localization in one dimension, with the random Schrödinger operator [71]

$$
\mathcal{H}_{\omega}=-\nabla^{2}+V_{0}(x)+\lambda V_{\omega}(x),
$$

where $\lambda>0, V_{0}(x)$ is a bounded potential periodic in $x$, and

$$
V_{\omega}(x)=\sum_{n=-\infty}^{\infty} \eta_{n}^{\omega} U\left(x-x_{n}\right) .
$$

In (23) $n$ runs over the integers, $U\left(x-x_{n}\right)$ is a nonegative localized potential centered at lattice site $x_{n}$, and the $\eta_{n}$ are i.i.d. random variables uniformly distributed in $[0,1]$.

Operators of the type given by (22) are known to have certain properties - see, e.g. [72]. If one first chooses a specific energy, then for a.e. realization $V_{\omega}$ of the random potential, that energy is part of its continuous spectrum (i.e., is not an eigenvalue of $\mathcal{H}_{\omega}$ ). On the other hand, if one first chooses a specific realization $V_{\omega}$, then there is almost surely a (countable) dense set of eigenvalues (in some appropriate energy interval) of $\mathcal{H}_{\omega}$, and of course this set depends on $\omega$.

Returning to the EA model in (say) three dimensions, it is amusing to speculate that a phenomenon like that described above for localization might occur for EA spin glasses, as follows: a) For every arbitrarily chosen $T$, there is a unique Gibbs state for a.e. $\mathcal{J}$; but b) there exists a (deterministic) $T_{c}$, such that for a.e. $\mathcal{J}$, the set of temperatures $T$ such that there are multiple Gibbs states (e.g., with $q_{E A} \neq 0$ ) for that $\mathcal{J}$ is dense in the entire temperature interval $\left[0, T_{c}\right]$. By an application of Fubini's theorem [73], property a) would necessarily imply that the set of such $T(\mathcal{J})$ 's would have zero Lebesgue measure in the temperature line.

Acknowledgments. This research was partially supported by NSF Grants DMS-01-02587 (CMN) and DMS-01-02541 (DLS).

\section{References}

[1] D. Sherrington and S. Kirkpatrick, Phys. Rev. Lett. 35, 1792 (1975).

[2] S. Edwards and P.W. Anderson, J. Phys. F 5, 965 (1975). 
[3] G. Parisi, Phys. Rev. Lett. 43, 1754 (1979).

[4] G. Parisi, Phys. Rev. Lett. 50, 1946 (1983).

[5] M. Mézard, G. Parisi, N. Sourlas, G. Toulouse, and M. Virasoro, Phys. Rev. Lett. 52, 1156 (1984).

[6] M. Mézard, G. Parisi, N. Sourlas, G. Toulouse, and M. Virasoro, J. Phys. (Paris) 45, 843 (1984).

[7] We gloss over here the serious problems surrounding the definition of pure states in the SK model. See the companion paper in this volume [21] for a more thorough discussion.

[8] C.M. Newman and D.L. Stein, J. Phys.: Condensed Matter 15, R1319 (2003).

[9] C.M. Newman and D.L. Stein, J. Stat. Phys. 106, 213 (2002).

[10] H.O. Georgii, Gibbs Measures and Phase Transitions (de Gruyter Studies in Mathematics, Berlin, 1988).

[11] D. Ruelle, Statistical Mechanics (Benjamin, New York, 1969).

[12] O. Lanford, in Statistical Mechanics and Mathematical Problems, Lecture Notes in Physics, v. 20 (Springer, 1973), pp. 1-135.

[13] B. Simon, The Statistical Mechanics of Lattice Gases v. 1, (Princeton Univ. Press, Princeton, 1993).

[14] J. Slawny, in C. Domb and J.L. Lebowitz (eds.), Phase Transitions and Critical Phenomena v. 11 (Academic Press, London, 1986), pp. 128-205.

[15] R.L. Dobrushin and S.B. Shlosman, in S.P. Novikov (ed.), Soviet Scientific Reviews C. Math. Phys., v. 5 (Harwood Academic Publ.,1985), pp. 53-196.

[16] A.C.D. van Enter and J.L. van Hemmen, Phys. Rev. A 29, 355 (1984).

[17] K. Binder and A.P. Young, Rev. Mod. Phys. 58, 801 (1986).

[18] M. Mézard, G. Parisi, and M.A. Virasoro, Spin Glass Theory and Beyond (World Scientific, Singapore, 1987).

[19] C.M. Newman and D.L. Stein, Phys. Rev. B 46, 973 (1992).

[20] C.M. Newman and D.L. Stein, Phys. Rev. Lett. 76, 4821 (1996).

[21] C.M. Newman and D.L. Stein, Local vs. Global Variables for Spin Glasses, to appear in 2004 Ascona workshop proceedings.

[22] C.M. Newman and D.L. Stein, in Mathematics of Spin Glasses and Neural Networks, ed. A. Bovier and P. Picco (Birkhäuser, Boston, 1997), pp. 243-287.

[23] C.M. Newman and D.L. Stein, Phys. Rev. E 55, 5194 (1997).

[24] C.M. Newman and D.L. Stein, Phys. Rev. E 57, 1356 (1998).

[25] M. Aizenman and J. Wehr, Commun. Math. Phys. 130, 489 (1990). 
[26] F. Krzakala and O.C. Martin, Phys. Rev. Lett. 85, 3013 (2000).

[27] M. Palassini and A.P. Young, Phys. Rev. Lett. 85, 3017 (2000).

[28] C.M. Newman and D.L. Stein, Phys. Rev. Lett. 87, 077201 (2001).

[29] C.M. Newman and D.L. Stein, Annales Henri Poincare`4 4, Suppl. 1, S497 (2003).

[30] A.T. Ogielski, Phys. Rev. B 32, 7384 (1985).

[31] A.T. Ogielski and I. Morgenstern, Phys. Rev. Lett. 54, 928 (1985).

[32] N. Kawashima and A.P. Young, Phys. Rev. B 53, R484 (1996).

[33] M.E. Fisher and R.R.P. Singh, in Disorder in Physical Systems, edited by G. Grimmett and D.J.A. Welsh (Clarendon Press, Oxford, 1990), pp. 87-111.

[34] M.J. Thill and H.J. Hilhorst, J. Phys. I 6, 67 (1996).

[35] C.M. Newman and D.L. Stein, Phys. Rev. Lett. 84, 3966 (2000).

[36] C.M. Newman and D.L. Stein, Phys. Rev. E 63, 16101-1 (2001).

[37] W.L. McMillan, J. Phys. C 17, 3179 (1984).

[38] A.J. Bray and M.A. Moore, Phys. Rev. B 31, 631 (1985).

[39] A.J. Bray and M.A. Moore, Phys. Rev. Lett. 58, 57 (1987).

[40] D.S. Fisher and D.A. Huse, Phys. Rev. Lett. 56, 1601 (1986).

[41] D.A. Huse and D.S. Fisher, J. Phys. A 20, L997 (1987).

[42] D.S. Fisher and D.A. Huse, J. Phys. A 20, L1005 (1987).

[43] D.S. Fisher and D.A. Huse, Phys. Rev. B 38, 386 (1988).

[44] C.M. Newman and D.L. Stein, Phys. Rev. Lett. 76, 515 (1996).

[45] S. Franz, G. Parisi, and M.A. Virasoro, J. Phys. I (France) 4, 1657 (1994).

[46] E. Marinari, G. Parisi, F. Ricci-Tersenghi, J.J. Ruiz-Lorenzo, and F. Zuliani, J. Stat. Phys. 98, 973 (2000).

[47] C.M. Newman and D.L. Stein, Phys. Rev. Lett. 72, 2286 (1994).

[48] C.M. Newman and D.L. Stein, J. Stat. Phys. 82, 1113 (1996).

[49] J.R. Banavar, M. Cieplak, A. Maritan, Phys. Rev. Lett. 72, 2320 (1994).

[50] S. Franz, M. Mézard, G. Parisi, and L. Peliti, Phys. Rev. Lett. 81, 1758 (1998).

[51] P.W. Kasteleyn and C.M. Fortuin, J. Phys. Soc. Jpn. 26, 11 (1969). 
[52] C.M. Fortuin and P.W. Kasteleyn, Physica 57, 536 (1972).

[53] A. Gandolfi, M.S. Keane, and C.M. Newman, Prob. Thy. Rel. Fields 92, 511 (1992).

[54] R.H. Swendsen and J.S. Wang, Phys. Rev. Lett. 58, 86 (1987).

[55] Y. Kasai and A. Okiji, Prog. Theor. Phys. 79, 1080 (1988).

[56] C. Newman, in Probability and Phase Transitions, edited by G. Grimmett (Kluwer, Dordrecht, 1993), pp. 247-260.

[57] G.R. Grimmett, Percolation (Springer, Berlin, 1999).

[58] C.M. Fortuin, P.W. Kasteleyn, and J. Ginibre, Commun. Math. Phys. bf 22, 89 (1971).

[59] T.E. Harris, Proc. Camb. Phil. Soc. 56, 13 (1960).

[60] M.F. Sykes and J.W. Essam, Phys. Rev. Lett. 10, 3 (1963).

[61] J. Wierman, Adv. Appl. Prob. 13, 293 (1981).

[62] J.M. Kosterlitz and D.J. Thouless, J. Phys. C 6, 1181 (1973).

[63] J.R. Banavar and A.J. Bray, Phys. Rev. B 35, 8888 (1987).

[64] I. Kondor, J. Phys. A, Math. Gen. 22, L163 (1989).

[65] M. Ney-Nifle and H.J. Hilhorst, Physica A 193, 48 (1993).

[66] F. Ritort, Phys. Rev. B 50, 6844 (1994).

[67] A. Billoire and E. Marinari, J. Phys. A, Math. Gen. 33, L265 (2000).

[68] J-P. Bouchaud, V. Dupuis, J. Hammann, and E. Vincent, Phys. Rev. B 65, 024439 (2002).

[69] A. Billoire and E. Marinari, cond-mat/0202473

[70] T. Rizzo and A. Crisanti, Phys. Rev. Lett. 90, 137201 (2003).

[71] M. Aizenman, A. Elgart, S. Naboko, J.H. Schenker, and G. Stolz, math-ph/0308023

[72] R. Carmona and J. Lacroix, Spectral Theory of Random Schrödinger Operators (Birkhäuser, Boston, 1990).

[73] W. Feller, An Introduction to Probability Theory and Its Applications, Vol. II (Wiley, NY, 1971), p. 124. 